\title{
Basic Science
}

\section{e Evaluation of Neuropathic Pain in a Rat Model of Total Brachial Plexus Avulsion from Behavior to Brain Metabolism}

Jun Shen, MD1, Bei-Bei Huo, MD1, Mou-Xiong Zheng, MD, PhD ${ }^{1,3}$, Xu-Yun Hua, MD, PhD ${ }^{1,3}$, Hao Shen, MD, PhD ${ }^{4}$, Ye-Chen Lu, MD', Dong-Lang Jiang, $\mathrm{MD}^{5}$, Chun-Lei Shan, MD, PhD ${ }^{1,2}$, and Jian-Guang $\mathrm{Xu}, \mathrm{MD}, \mathrm{PhD}^{1,2}$

From: 'School of Rehabilitation Science, Shanghai University of Traditional Chinese Medicine Shanghai, China; ${ }^{2}$ Department of Rehabilitation Medicine, Yueyang Hospital, Shanghai University of Traditional Chinese Medicine Shanghai, China; ${ }^{3}$ Department of Trauma and Orthopedics, Yueyang Hospital, Shanghai

University of Traditional Chinese Medicine, Shanghai, China;
4Department of Orthopaedics, Xinhua Hospital Affiliated to Shanghai Jiaotong University School of Medicine, Shanghai 200092, PR China; ${ }^{5}$ Department

of Nuclear Medicine, Huashan Hospital, Shanghai Medical College, Fudan University, Shanghai, China

Address Correspondence: Jian-Guang Xu, MD, PhD School of Rehabilitation Science Shanghai University of Traditional Chinese Medicine

No. 1200 Cailun Rd., Shanghai 201203, China

E-mail: xjg@shutcm.edu.cn

Disclaimer: JS and BBH equally contributed to this work. This work was supported by Shanghai Education Committee [Grant No.

A2-P1600325]; Shanghai Science and Technology Committee [Grant No.: 18511108300 18441903900; 18441903800]; Graduate innovation ability project of Shanghai University of Traditional Chinese

Medicine[Grant No.:E4-Di7017]. Conflict of interest: Each author certifies that he or she, or a

member of his or her immediate family, has no commercial

association (i.e., consultancies, stock ownership, equity interest, patent/licensing arrangements etc.) that might pose a conflict of

interest in connection with the submitted manuscript.

Manuscript received: 07-10-2018 Revised manuscript received: 09-22-2018 Accepted for publication: 11-05-2018

Free full manuscript: www.painphysicianjournal.com
Background: Approximately $30 \%$ to $80 \%$ of patients with brachial plexus avulsion (BPA) developed neuropathic pain. It is an intolerable neuropathic pain, which brings heavy burden to family and society. In addition to motor and sensory deficits, neuropathic pain can be another serious sequela that equally influences the patient. The development of a microsurgical technique has promoted the treatment and rehabilitation of brachial plexus injury, but pain relief after BPA is still a difficult problem.

Objectives: The present study aimed to semi-quantify changes in the behavior, spinal cord and cerebral metabolism in a neuropathic pain model following BPA injury in rats.

Study Design: Controlled animal study.

Setting: Institute of Rehabilitation Medicine, Shanghai, China.

Methods: A total of 15 Sprague-Dawley rats, weighing 200 to 220 g, were randomly divided into 2 groups: experimental group $(n=10)$ and control group $(n=5)$. In the experimental group, neuropathic pain induced by BPA was established by directly avulsing the $C 5, C 6, C 7, C 8$, and $\mathrm{T} 1$ roots on the right side from the spinal cord. Rats in the control group only received openclose surgery. The autotomic behavior of biting their own digits was recorded and scored at 2 months after the surgery. Small animal positron emission tomography/computed tomography (PET/CT) images after injection of a 2-[18 $\mathrm{F}]$-fluoro-2-deoxy-D-glucose ( $\left.{ }^{18} \mathrm{~F}-\mathrm{FDG}\right)$ tracer were acquired to evaluate glucose metabolism in pain-related brain regions before and after the surgery, respectively. Semi-quantitative values of cortical to cerebellum standardized uptake value (SUV) ratios were calculated. Then, the animals were euthanized and the cervical segments of the spinal cord were removed for detection of glial fibrillary acidic protein (GFAP) expression in the astrocytes by immunohistochemical assay.

Results: Nine of the 10 rats (90\%) in the experimental group showed autotomic behavior at 2 months after the surgery. Slight autotomic behavior was noted only in one of 5 rats (20\%) from the control group. The autotomic score in the experimental group was significantly higher than that in the control group $(5.4 \pm 1.0$ vs. $0.2 \pm 0.4, P<0.05)$. The experimental group showed significantly higher SUV ratio in both the right and left thalamus, compared to the control group $(P<0.05)$. Immunohistochemical assay demonstrated that GFAP positive astrocytes in the dorsal horn at the injured side significantly increased compared to the control group $(P<0.05)$.

Limitations: There are differences between small animals and human beings, and the structure and function of the human brain is more complex than in rodents. Therefore, extrapolation of the present conclusion should be cautious.

Conclusions: The present study reported a unique model of neuropathic pain following total BPA in rodents, which was demonstrated by a higher rate and score of autotomic behavior. More astrocytes were found activated in the spinal cord at the corresponding level of C5 and C6 spinal cord. In the small animal PET/CT imaging, significantly higher standardized glucose 
metabolic activity was found in both the right and left thalamus in the experimental group. The present study semi-quantified the neuropathic pain behavior in rats and explored the plastic changes in the spinal and brain metabolism.

Key words: Brachial plexus avulsion, small animal PET/CT, glucose metabolism, neuropathic pain, astrocyte, ${ }^{18} \mathrm{~F}-\mathrm{FDG}$

Pain Physician 2019: 22:E215-E224

D) rachial plexus avulsion (BPA), which leads to complete or partial paralysis of the ipsilateral upper limbs, is one of the most serious types of peripheral nerve injury (1). Brachial plexus injuries in adults results in devastating consequences on the upper extremity function and life quality (2). When treating BPA, it is hard to re-establish reinnervation of all the muscles in the paralyzed upper limb. Even with successful surgical reconnection of the injured brachial plexus, the death of the motoneuron body often results in unsatisfactory outcomes. Structures at multiple levels are involved following BPA, from the axons, neurons, and spinal cord, to the subcortical and cortical structures (3).

In addition to motor and sensory deficits, neuropathic pain can be another serious sequela that influences the patient (4-6). The incidence rates of neuropathic pain following BPA varies among different studies (7-9). Approximately $30 \%$ to $80 \%$ of patients with BPA developed neuropathic pain (10). The main characteristics of neuropathic pain following BPA are the rapid onset of pain (an effect that occurs immediately after injury) and the long-lasting development of neuropathy, which may be evidenced even distant from the site of the lesion (11). Neuropathic pain would also lead to mechanical allodynia and cold allodynia. It is now acknowledged that alterations in both the peripheral and central nervous systems are involved in the genesis of neuropathic pain $(3,12)$. Several studies reported that glial cells were significantly activated in the spinal cord at the injury level of BPA (13). The activation of microglia and astrocytes were associated with initiation and persistence of pain status, which lasted 3 months following BPA (14). Therefore, this phenomenon was potentially related to the existence of neuropathic pain.

In vivo positron emission tomography/computed tomography (PET/CT) imaging can be helpful in exploring the brain activity in neuropathic pain following BPA. A frequently used tracer to quantify the amount of glucose consumption in the brain because of its relatively long half-life period (110 minutes) is $2-\left[{ }^{18} \mathrm{~F}\right]$ -
fluoro-2-deoxy-D-glucose ( $\left.{ }^{18} \mathrm{~F}-\mathrm{FDG}\right)$ (15). Higher accumulation of $18 \mathrm{~F}$ glucose indicates higher metabolic activity in the brain (16). Therefore, the level of glucose used is significantly associated with the degree of neuronal activity (17).

Small animal ${ }^{18} \mathrm{~F}-\mathrm{FDG}$ PET/CT imaging has already been used to investigate whole-body metabolic activity and acquire images reflecting quantitative metabolic information in regions of interest (ROI) in different diseases (18). In the present study, the PET/CT was applied to evaluate BPA-induced neuropathic pain in adult rats. In addition, pathological changes in the corresponding spinal segments were also obtained.

In conclusion, the present study aimed at quantifying the behavior changes in neuropathic pain following BPA and exploring alterations both in the spinal cord and brain metabolism.

\section{Methods}

\section{Animal Model}

A total of 15 female Sprague-Dawley rats weighing from 200 to $220 \mathrm{~g}$ and aged from 8 to 10 weeks were used in this study. The rats were randomly divided into 2 groups: the experimental group $(n=10)$ received total BPA on the right side, and the control group ( $n=$ 5 ) only received open-close surgery (sham surgery) on the same side. The rats were obtained from Shanghai Slack Laboratory Animal Limited Liability Company (Shanghai, China) and raised under the following environmental conditions: 12 hour/12 hour light/dark cycle, $20^{\circ} \mathrm{C}$ to $22^{\circ} \mathrm{C}$, plenty of food, and enough water. They were kept at least one week before any surgery or examination was conducted.

All protocols and procedures of animal use were carried out according with the Guide for the Care and Use of Laboratory Animals described by the U.S. National Institutes of Health. The authors declared that all the experiments were approved by the Animal Ethical Committee of Shanghai University of Traditional Chinese Medicine before the study began and followed the approved guidelines. The researchers made every 
effort to minimize the suffering of the animals and the number of animals used.

The rats in the experimental group were anaesthetized with sodium pentobarbital by intraperitoneal injection (40 mg/kg), and then placed on a heated clean surgical table. Along the dorsal midline from the occiput to scapular angulus superior, an approximate $4-\mathrm{cm}$ incision was made using $C 7$ vertebrae as bone marks. The longissimus capitis muscle, semispinal muscle of neck, biventer cervicis, and complex muscle were identified and retracted. Brachial plexus roots of the right side were accessed through the posterior approach under an operating microscope (magnification $\times 10$ ). After the muscles on the vertebral plate and the spinal process were removed, hemilaminectomies from C4 to T1 were performed to expose the nerve roots from $\mathrm{C} 5$ to $\mathrm{T} 1$. When the roots had been clearly dissociated, they were avulsed quickly from the spinal cord by a retractor. Penicillin powder was used for preventing incisional infection. All operations were performed by the same experimenter.

\section{Autotomic Behavior Assessment}

The autotomic behavior of all rats were recorded at 2 months after the surgery. The autotomic score was assessed according to the scale reported by Wall et al (19). Briefly, one point was recorded if 2 or more nails were injured. Every one point was added when every half of the digits was involved. An accumulated score was recorded as the final result. The total score was no more than 13 points.

\section{Small Animal PET/CT Imaging}

Small animal PET/CT imaging was performed on Siemens Inveon PET/CT scanners (Siemens Inc., Chicago, USA) with an LSO crystal detector. The reconstructed spatial resolution was $2.0 \mathrm{~mm}$ at the center of the field of view (FOV), and the axial and transaxial FOVs were 10.0 and $12.7 \mathrm{~cm}$, respectively (20). The ${ }^{18} \mathrm{~F}-\mathrm{FDG}$, with an activity of $500 \mathrm{Ci} / \mathrm{mmol}$, was prepared in the Center at the Department of Nuclear Medicine, Huashan Hospital, Fudan University. A dose of $0.5 \mathrm{mCi}$ of pyrogen-free 18F-FDG was injected through the tail vein and the rats were returned to their home cage in a room with minimal ambient noise for the uptake period (21). Then, the rats were allowed to move around for 40 minutes after tracer injection to reduce the influence of the anesthesia on brain glucose metabolism (22). Subsequently, the animals were anaesthetized by intraperitoneal injection of $40 \mathrm{mg}$ per $1000 \mathrm{~g}$ of body weight of sodium pentobarbital and placed prostate in the small animal PET/
CT scanner with limbs extended. A 10-minute static PET acquisition was performed followed by a 7-minute anatomic CT scan acquisition. Data were collected and reconstructed by a maximum a posteriori probability algorithm with a pixel size of $0.776 \times 0.776 \times 0.796$ $\mathrm{mm}^{3}$. Each PET image was spatially normalized into the space of an 18F-FDG template using brain normalization in PMOD software (PMOD Beta Version 3.3, PMOD Technologies, Zurich, Switzerland) (23). Axial CT images were used to assign ROI per slice according to the rat's brain anatomy atlas (24). To quantify the alterations in cerebral glucose uptake in a spatiotemporal manner, a volume of interest brain atlas was used to determine the pre- and postoperative metabolic level. This procedure was accomplished by using the PMOD software package in conjunction with the Schiffer's rat brain template and atlas. Small animal PET/CT imaging was performed on Day 0 and Day 7 following BPA, respectively (Fig. 1).

\section{Analysis of PET/CT}

After the data collection, the PMOD software was rebuilt, then the thalamus area of the same side and the contralateral side was selected, and the standardized uptake value (SUV) was obtained. Compared with the SUV of the cerebellum, the relative ROI semiquantitative values of the brain region were obtained. Changes in the ROls were also recorded.

\section{Immunohistochemistry}

The rats were anesthetized by $1 \%$ sodium pentobarbital solution injected intraperitoneally at 8 weeks after the operation. They were perfused with $0.9 \%$ saline solution, followed by $500 \mathrm{~mL}$ of $4 \%$ paraformaldehyde in phosphate buffer transcardially. The dorsal horns of C5 and C6 spinal cord were resected and fixed in 10\% neutral buffered formalin solution. They were processed to prepare 3-mm thick paraffin sections. Immunohistochemistry was performed using a 3-step indirect process based on the labelled peroxidase complex method. Sections were rehydrated in descending grades of alcohol. Following blocking of endogenous peroxidase activity with $3 \% \mathrm{H}_{2} \mathrm{O}_{2}$ in methanol and nonspecific binding sites with 5\% BSA, the sections were incubated overnight at $4^{\circ} \mathrm{C}$ with a $1: 100$ dilution of rabbit antibody to glial fibrillary acidic protein (GFAP) (Abcam, Cambridge, MA) and a rabbit antibody to Iba1 (Abcam). Then, biotinylated secondary antibody was added at a concentration of $2 \%$ for 1 hour $\left(37^{\circ} \mathrm{C}\right)$ followed by addition of 4',6-diamidino2-phenylindole (DAPI) for 5 minutes. Visualization of 
the reaction was performed using 3,3-diaminobenzidine as the chromogen, which produces a dark brown precipitate that is readily detected by light microscopy. The sections were then counterstained with Mayer's hematoxylin, dehydrated in ascending grades of alcohol, and cleared in xylene. Finally, we examined the sections using a microscope at 400x magnification. The sections were characterized quantitatively by digital image analysis using the Imagine-Pro Plus (Media Cybernetics Inc., Rockville, MD).Mean integrated optical density was applied to assess the staining intensity from the sections.

\section{Statistical Analysis}

Data were entered and analyzed with statistical software SPSS version 22.0 (IBM Corporation, Armonk, $\mathrm{NY}$ ). Results were presented as mean \pm standard deviation. The independent Student $t$ test was used in comparison between 2 groups when the data were normally distributed, whereas the Wilcoxon rank-sum test was used in non-normal distributed data. The paired $t$ test was used for within-subject comparisons. One-way analysis of variance was used for comparison of 2 groups or more. Two-tailed $P<0.05$ was considered statistically significant.

\section{Results}

\section{Autotomic Behavior}

At baseline, the average autotomic score and incidence rate of autotomic behavior in all limbs were 0 in both the experimental and control groups (Table 1). At the second month following right BPA in the experimental group ( $n=10$ ), autotomic phenomenon could be noted in all 4 limbs. The incidence rate of autotomic behavior was the highest in the right forelimb (90\%), whereas the incidence of autotomic phenomenon in the other 3 limbs was lower (left forelimb, 40\%; left hindlimb, 30\%; right hindlimb 50\%). Although the mean score was the highest in the right (injured) forelimb, there were no statistical differences between the left (intact) and the right (injured) sides. Higher autotomic score and incidence rate were detected in the right (injured) forelimb compared to the right forelimb in the control group $(n=5)$ at the second month following surgery $(P<0.05)$ (Fig. 1E, Table 1$)$.

\section{PET/CT Imaging}

The SUV of bilateral thalamus and cerebellum were calculated. SUV of the cerebellum was selected as
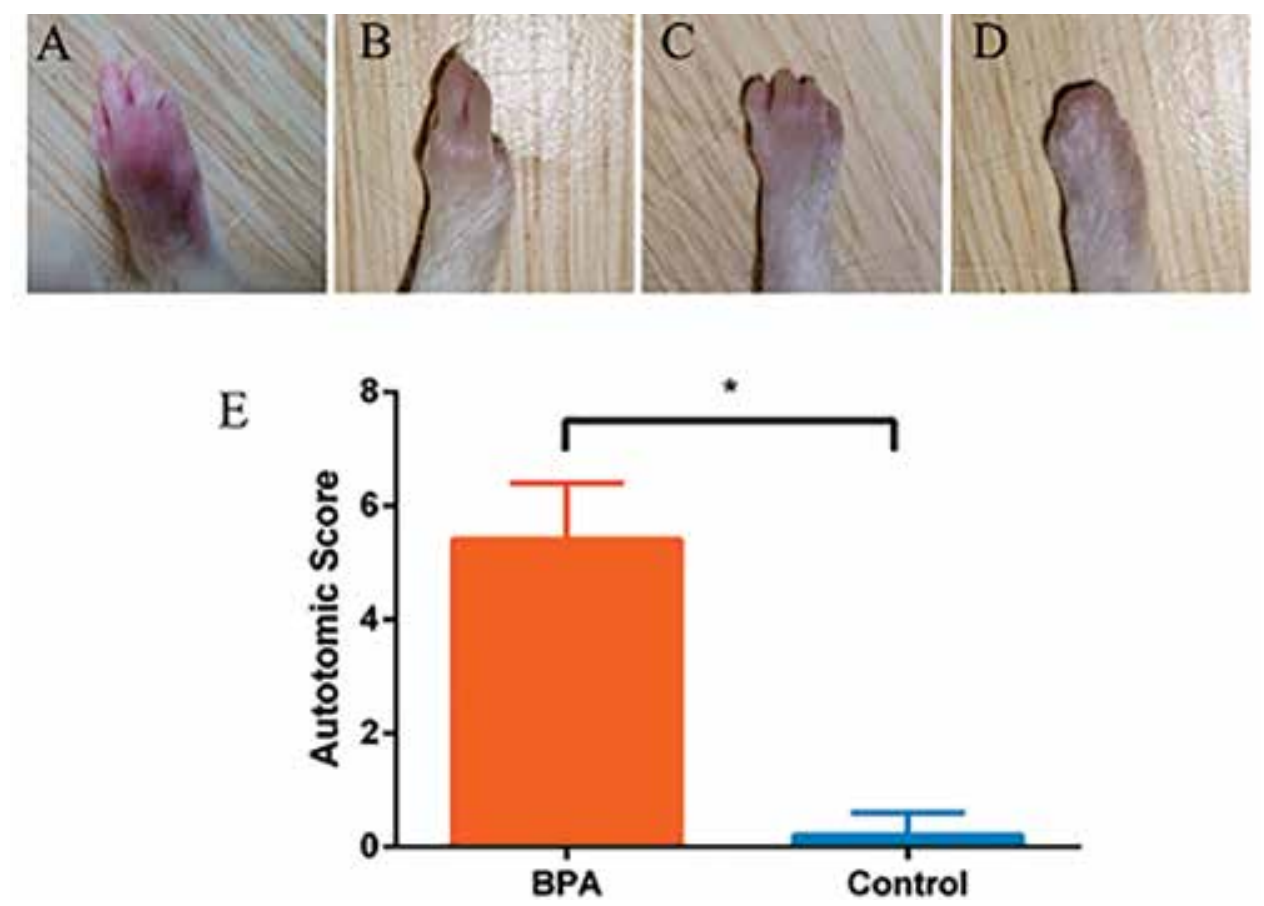

Fig. 1. Demonstration of autotomic behavior following total BPA and autotomic score between the experimental and control groups. (A) Autotomic behavior with only nails involved. (B) Autotomic behavior with one digit involved. (C,D) Autotomic behavior with more than one digit involved. (E) The histogram shows significantly higher autotomic score at the second postoperative month following total $B P A$ (experimental group, $n=10)$ than that following sham surgery (control group, $n=5$ ) $(P<0.01)$. 
Table 1. The average autotomic score and incidence rate of autotomic behavior in the experimental group (right BPA surgery) and control group (sham surgery).

\begin{tabular}{|c|c|c|c|c|}
\hline & \multicolumn{2}{|c|}{ Forelimb } & \multicolumn{2}{|c|}{ Hindlimb } \\
\hline & Left & Right & Left & Right \\
\hline \multicolumn{5}{|l|}{ Experimental group $(n=10)$} \\
\hline Baseline & $0(0 \%)$ & $0(0 \%)$ & $0(0 \%)$ & $0(0 \%)$ \\
\hline Second month following BPA & $3.5 \pm 4.6(40 \%)$ & $5.4 \pm 1.0(90 \%)$ & $3.8 \pm 4.9(30 \%)$ & $4.4 \pm 4.9(50 \%)$ \\
\hline \multicolumn{5}{|l|}{ Control group $(n=5)$} \\
\hline Baseline & $0(0 \%)$ & $0(0 \%)$ & $0(0 \%)$ & $0(0 \%)$ \\
\hline Second month following BPA & $0(0 \%)$ & $0.2 \pm 0.4(20 \%)$ & $0(0 \%)$ & $0(0 \%)$ \\
\hline
\end{tabular}

The number preceding the brackets indicates average autotomic scores (mean \pm standard deviation). The percentage within the brackets indicates the incidence rate of autotomic behavior in the group.

a reference and the results in bilateral thalamus were presented as SUV ratios (Fig. 2). The SUV ratio of bilateral thalamus were both significantly higher at the second month following BPA (experimental group) than that following sham surgery (control group) $(P<0.01)$ (Fig. 3).

\section{Immunohistochemistry}

At the second postoperative month, the number of GFAP positive cells of the dorsal horn following total BPA (experimental group) was significantly higher on the right (injured) side than that on the left (intact) side (Fig. 4). The number of GFAP positive cells in the right (injured) dorsal horn of the spinal cord in the experimental group was also significantly higher than that in the control group. No significant difference was found between the GFAP positive cells in the left (intact) dorsal horn of the spinal cord in the experimental group and that in the control group ( $P>0.05$ ) (Fig. 4E).

\section{Discussion}

Brachial plexus injury is one of the most devastating peripheral injuries. It includes 2 main types: preganglionic injury and postganglionic injury. The postganglionic brachial plexus injury model could be established by cutting off the brachial plexus nerves outside the intervertebral foramen.

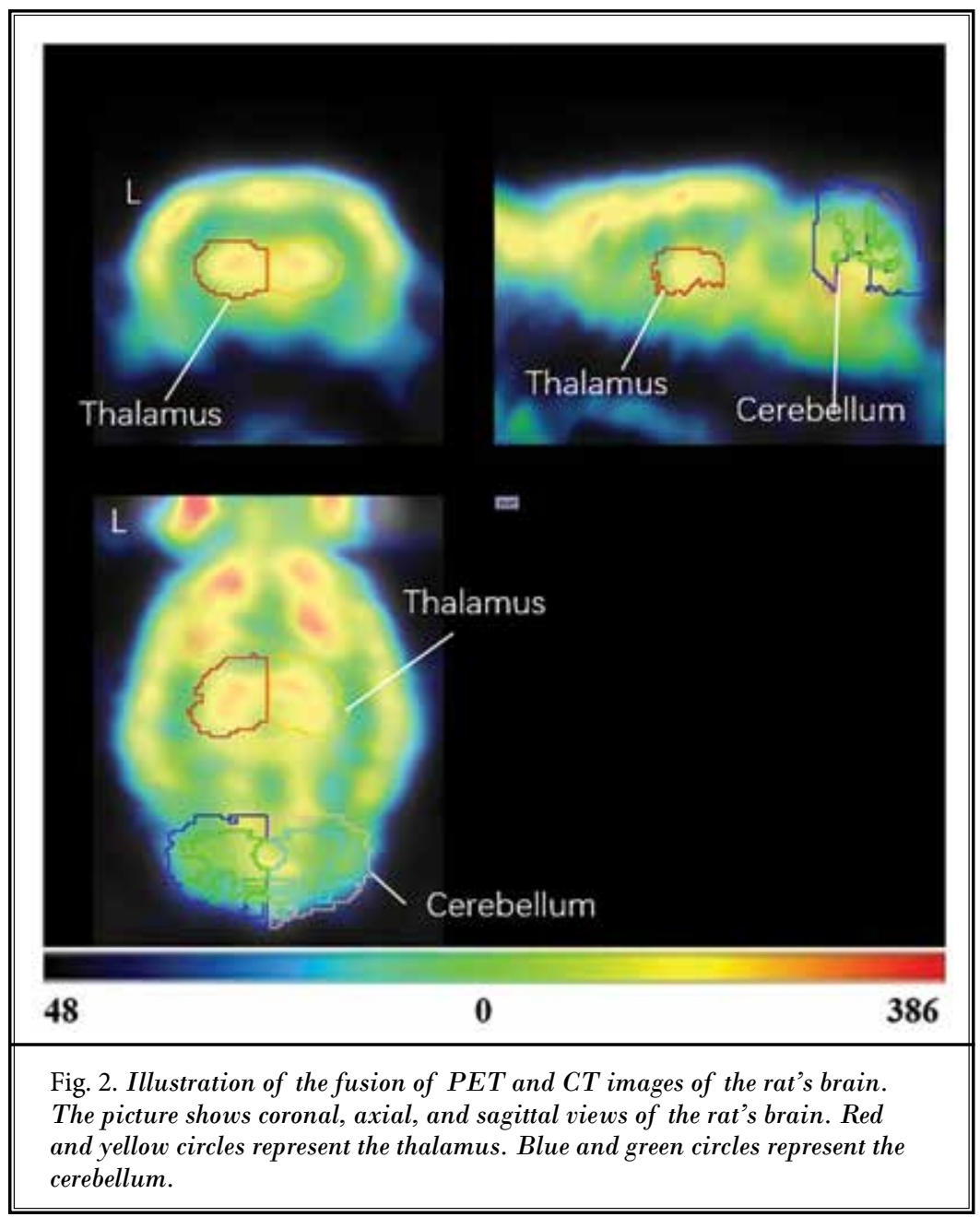

However, that is unsuitable to simulate the postganglionic injury. There are remarkable differences in the pathogenesis and pathological state between these 2 types of injuries. The preganglionic brachial plexus in- 

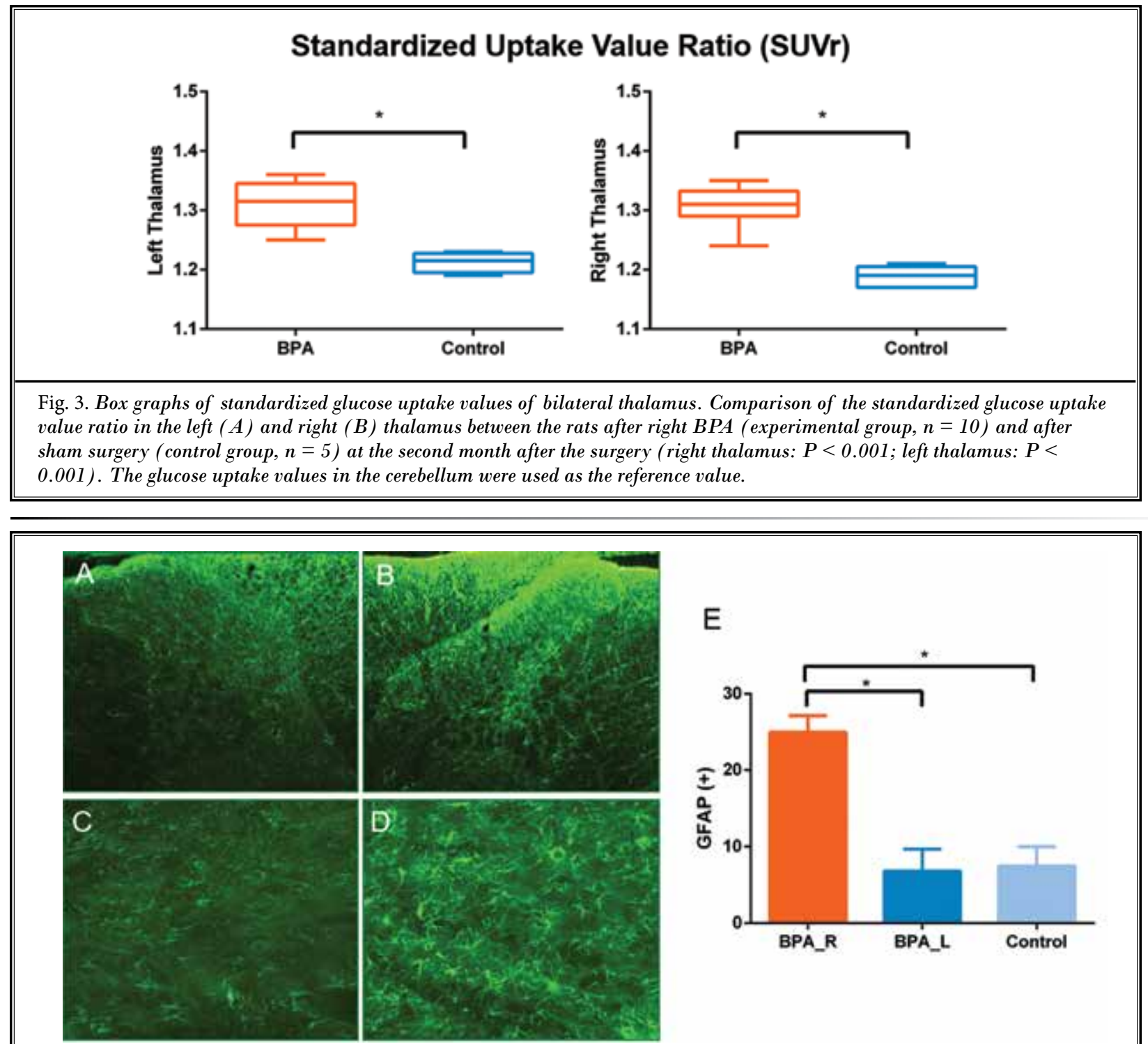

Fig. 4. Immunohistochemistry of GFAP in the spinal cord of cervical enlargement segment. (A,B) The GFAP staining of the dorsal horn on the left side (intact) at the second postoperative month following total BPA on the right side in the experimental group. (C,D) The GFAP staining of the dorsal horn on the right side (injured) at the second postoperative month following total $B P A$ on the right side in the experimental group. (E) Comparison of GFAP positive cells among bilateral sides of the spinal cord in the experimental and control groups. BPA_L: left dorsal horn of the spinal cord in the BPA group; BPA_R: right dorsal horn of the spinal cord in the BPA group.

jury is usually achieved by tearing the nerves from the spinal cord. The success rate of establishing a neuropathic pain model by tearing the brachial plexus nerves from outside the intervertebral foramen might not be satisfactory.

There have been other studies reporting neuropathic pain induced by BPA (25-34). However, in previ- ous studies, surgeries were performed via a horizontal incision parallel to the clavicle, and the brachial plexus nerves were exposed through a supraclavicular or subclavicular approach. The whole brachial plexus, or the lower trunk, was grasped with forceps and extracted from the spinal cord by traction. In these approaches, the clavicle should be retracted to expose the whole 
brachial plexus nerves, especially the lower trunk. As the lower trunk was close to the subclavian vessels anatomically, it was easy to cause bleeding while exploring the plexus, and it was difficult to confirm whether the BPA was a preganglionic or postganglionic type. In the present study, a posterior approach was used to establish the BPA model in rats. Laminectomy was performed to ensure a clear exposure of all the $\mathrm{C} 5, \mathrm{C} 6, \mathrm{C} 7, \mathrm{C} 8$, and $\mathrm{T} 1$ nerve roots, and the brachial plexus on the right side were completely extracted from the spinal cord under direct vision. The accuracy and success rate could be more stable in this approach. Despite more extensive injury caused by this approach, no rat in the experimental group died as a direct result of the surgical procedure. No rat presented foraging or abnormal activity except for upper limb paralysis and self-disability. Significantly higher autotomic score was recorded in the experimental group, which implied a higher incidence rate of neuropathic pain. This indicated that the posterior approach used in this study was a feasible method to establish the neuropathic pain model.

Autotomic behavior is one of the characters of neuropathic pain after nerve injury (35). Following the injury of sciatic nerve, for example, the rats showed scratching and biting of the injured limb when applying thermal or chemical stimulations on it. In particular, when we clamped the limb with forceps, the rat would also attack the forceps (36). Therefore, autotomic behavior is considered a reflection to neuropathic pain, which is quite similar to the clinical performance in humans (29). Sensory deafferentation was one of the possible reasons, however, researchers did not find the evidence of autotomic behavior after blockade of the sciatic nerve with lidocaine in rats (37). Therefore, the pathological process underlying this autotomic behavior is complicated and may involve multiple mechanisms.

In the present study, a total of $90 \%$ of rats showed autotomic behavior after BPA, compared with $20 \%$ in the control group. According to the scale reported by Wall et al (19), the autotomic score in the experimental group was also significantly higher than that in the control group. It suggested that the neuropathic pain model established by BPA through the posterior approach was relatively reliable.

The GFAP is a unique kind of intermediate filament protein particularly expressed in the astrocytes. It is widely used as a marker for the astrocyte state of activity (30). In the spinal cord, astrocytes are mainly involved in maintaining homeostasis of the nervous system, including the concentration of ions and glutamate, the function of neurons, and the capacity of self-repair. In 1991, Garrison et al (38) for the first time proposed that activation of spinal glial cells was associated with pain sensitization. They found that the factors that caused pain sensitization could also activate astrocytes in the spinal cord. Drugs that block the sensitization of pain can also block the activation of astrocytes in the spinal cord $(38,39)$. Since then, various reports have found that in the conditions of inflammatory substance administration (such as formalin), peripheral nerve trauma, bone cancer, lumbar spinal nerve root ligation, spinal cord trauma, or activation of the immune system in the spinal cord, the resting spinal astrocytes would be activated and release a variety of pain mediators $(3,10,40,41)$ such as proinflammatory cytokines, interleukin-I $\beta$, tumor necrosis factor, interleukin-6, TGF- $\beta$, inflammatory mediators and neuroactive nitric oxide. These mediators can significantly inhibit or alleviate neuropathic pain by inhibiting secretion of inflammatory cytokines from glial cells or interfering with glial cell metabolism. Therefore, activation of spinal glial cells may be the key to chronic pain (42). The present study showed that the expression of GFAP in the spinal cord increased significantly following BPA, mainly in the I and II layers of spinal cord. Specifically, the astrocyte body increased in size and number, deepened in color, and the processes became thicker and longer. The GFAP (+) cells were dispersedly expressed in the control group as well as in the intact side of the spinal cord in the experimental group, but the body was less apparent, the dendrite was thinner, and the dye was lighter. The expression of GFAP on the injured side of the spinal cord in the experimental group was significantly different from that of the intact side or from the control group. Activation of the astrocytes may be involved in the maintenance and regulation of chronic neuropathic pain. It is also related with high incidence of autotomic behavior and severity of autotomic score in the experimental group.

With the rapid development of neuroimaging technology, we are now able to explore the central mechanism of neuropathic pain with noninvasive methods. Previous studies have suggested several brain regions closely related with neuropathic pain, including the primary sensory cortex (S1), the secondary sensory cortex (S2), the insula, the anterior cingulate cortex (ACC), the thalamus, and the prefrontal cortex (PFC) (43). These regions constitute network structure called pain matrix in the brain. Previous studies found that the primary and secondary sensory cortices, the lateral 
thalamus, and the posterior insula are associated with pain and sensory discrimination. The prefrontal gyrus and the anterior insula play an important role in the onset, development, and feedback of painful emotions, whereas prefrontal lobes are more involved in brain activity related to pain cognition at a higher level. According to the report by Peyron (44) on patients with neuropathic pain, general mechanical stimulation can induce severe pain in the skin of the affected side. Meanwhile, the somatosensory network in the mirror area of the contralateral hemisphere, the insular cortex, the S1 and M1 are also activated $(44,45)$. Studies reported that capsaicin-induced hyperalgesia would result in activation of the bilateral S2, lateral PFC, frontal and lower frontal gyrus $(43,46)$. Studies in pain-related attention found that ACC and frontal orbital gyrus are increased related to pain activity, whereas thalamic and insular activities are suppressed (47).

In the present study, the bilateral thalamus showed significantly increased metabolism. It reflected an increase in the activity of neurons in this area and might imply increased activity of pain information exchange. According to previous studies, the thalamus is related to neuropathic pain and could be a potential interventional target.

Generally, the thalamus is the most important transfer structure for sensory fibers in animals that have more evolved brains. The neurons in the thalamus receive projects from peripheral sensory fibers and then project to the cerebral cortex. The thalamus is responsible for primary processing of sensation, and it is connected with the hypothalamus and striatum, and these connected structures are the subcortical center of many complicated unconditioned reflexes (48). Researchers suggested that pain would be aggravated by activating the thalamus. Electrophysiological studies proved that the thalamus is sensitive to nociceptive afferent impulses (49). It is reported that damaged function or structure of the thalamus may be one of the reasons for neuropathic pain (50).

Chronic neuropathic pain is not simply a prolonged acute pain that protects us from potential and more severe injuries. Chronic pain, however, is maladaptive.
It is characterized by many positive symptoms, such as spontaneous pain, allodynia, and hyperalgesia. Complicated brain activities might be involved in the constant perception of pain in individuals with neuropathic pain $(32,33,48)$. Hyperexcitability of the thalamus is a possible reason for these enhanced activities. Meanwhile, there are bilateral projections to the thalamus from spinothalamic neurons in rats, which could explain an upregulation of metabolism in the bilateral thalamus $(51,52)$. However, the subregions of the thalamus were difficult to distinguish in the present study. Further research is still needed to demonstrate the role of the thalamus in the maintenance of neuropathic pain.

\section{Limitations}

There are differences between small animals and human beings, and the structure and function of the human brain is more complex than in rodents. Therefore, extrapolation of the present conclusion should be cautious.

\section{Conclusions}

The present study reported a unique neuropathic pain model in rats induced by complete avulsion of the brachial plexus nerves. Autotomic behavior related to neuropathic pain was semi-quantified and a significantly higher score was noted in the BPA group than that in the sham surgery group. More activated astrocytes were found in the dorsal horn of the spinal cord ipsilateral to the injured side, indicated by more positive GFAP cells. Higher metabolic activity was noted in the bilateral thalamus in the ${ }^{18} \mathrm{~F}-\mathrm{FDG}$ PET/CT imaging. We conclude that several levels of structures are involved in the mechanisms underlying initiation and persistence of neuropathic pain following BPA. Reorganization in the spinal cord and brain might both be involved in this process.

\section{Acknowledgment}

The authors ensure that all authors meet the criteria listed in the author guidelines. Each author had participated sufficiently in the work to take public responsibility for appropriate portions of the content. 


\section{References}

1. Zhou Y, Liu P, Rui J, Zhao X, Lao J. The clinical characteristics of neuropathic pain in patients with total brachial plexus avulsion: A 30-case study. Injury 2016; 47:1719-1724.

2. Bruxelle J, Travers V, Thiebaut JB. Occurrence and treatment of pain after brachial plexus injury. Clin Orthop Relat Res 1988; 87-95.

3. Teixeira MJ, Paz MGDS, Bina MT, Santos SN, Raicher I, Galhardoni R, Fernandes DT, Lin TY, Baptista AF, Andrade DCD. Neuropathic pain after brachial plexus avulsion-Central and peripheral mechanisms. BMC Neurol 2015; 15:73.

4. Tantigate D, Wongtrakul S, Vathana $\mathrm{T}$, Limthongthang R, Songcharoen $\mathrm{P}$. Neuropathic pain in brachial plexus injury. Hand Surg 2015; 20:39-45

5. Ochiai $N$, Nagano A, Sugioka $H$, Hara T. Nerve grafting in brachial plexus injuries. Results of free grafts in 90 patients. J Bone Joint Surg Br 1996; 78:754-758.

6. Price TJ, Basbaum Al, Bresnahan J, Chambers JF, De Koninck Y, Edwards RR, Ji RR, Katz J, Kavelaars A, Levine JD, Porter L, Schechter N, Sluka KA, Terman GW, Wager TD, Yaksh TL, Dworkin RH. Transition to chronic pain: Opportunities for novel therapeutics. Nat Rev Neurosci 2018 May 15. [Epub ahead of print].

7. Rodrigues-Filho R, Santos AR, Bertelli JA, Calixto JB. Avulsion injury of the rat brachial plexus triggers hyperalgesia and allodynia in the hindpaws: A new model for the study of neuropathic pain. Brain Res 2003; 982:186-194.

8. Parry CB. Pain in avulsion of the brachia plexus. Neurosurgery 1984; 15:960-965.

9. Bertelli JA, Taleb M, Saadi A, Mira JC, Pecotdechavassine $M$. The rat brachial plexus and its terminal branches: An experimental model for the study of peripheral nerve regeneration. Microsurgery 1995; 16:77-85.

10. Abdelaziz S, Ghaleb AH. Cervical spinal cord stimulation for the management of pain from brachial plexus avulsion. Pain Med 2014; 15:712-714.

11. Kauppila T. Correlation between autotomy-behavior and current theories of neuropathic pain. Neurosci Biobehav Rev 1998; 23:111-129.

12. Qiu YQ, Hua XY, Zuo CT, Li T, Zheng MX, Shen YD, Xu JG, Gu YD, Rossini PM, Xu WD. Deactivation of distant pain-related regions induced by 20 -day rTMS:
A case study of one-week pain relief for long-term intractable deafferentation pain. Pain Physician 2014; 17:E99-E105.

13. Ji RR, Chamessian A, Zhang YQ. Pain regulation by non-neuronal cells and inflammation. Science 2016; 354:572-577.

14. Ji RR, Berta T, Nedergaard M. Glia and pain: Is chronic pain a gliopathy? Pain 2013; 154:S10-S28.

15. Kornblum HI, Araujo DM, Annala AJ, Tatsukawa KJ, Phelps ME, Cherry SR. In vivo imaging of neuronal activation and plasticity in the rat brain by high resolution positron emission tomography (microPET). Nat Biotechnol 2000; 18:655-660.

16. Bustamante E, Pedersen PL. High aerobic glycolysis of rat hepatoma cells in culture: Role of mitochondrial hexokinase. Proc Natl Acad Sci U S A 1977; 74:3735-3739

17. Bruehl C, Witte OW. Cellular activity underlying altered brain metabolism during focal epileptic activity. Ann Neurol 1995; 38:414-420.

18. Chugani HT, Hovda DA, Villablanca JR, Phelps ME, Xu WF. Metabolic maturation of the brain: A study of local cerebral glucose utilization in the developing cat. J Cerebr Blood F Met 1991; 11:35-47.

19. Wall PD, Scadding JW, Tomkiewicz MM The production and prevention of experimental anesthesia dolorosa. Pain 1979; 6:175-182.

20. Parthoens J, Verhaeghe J, Wyckhuys $T$, Stroobants S, Staelens S. Small-animal repetitive transcranial magnetic stimulation combined with [18F]-FDG microPET to quantify the neuromodulation effect in the rat brain. Neuroscience 2014; 275:436-443.

21. Gao F, Wang S, Guo Y, Wang J, Lou M, Wu J, Ding $M$, Tian $M$, Zhang $H$. Protective effects of repetitive transcranial magnetic stimulation in a rat model of transient cerebral ischaemia: A microPET study. Eur ] Nucl Med Mol Imaging 2010; 37:954-961.

22. Matsumura A, Mizokawa SM, Wada Y, Nozaki S, Nakamura F, Shiomi S, Och $\mathrm{H}$, Watanabe Y. Assessment of microPET performance in analyzing the rat brain under different types of anesthesia: Comparison between quantitative data obtained with microPET and ex vivo autoradiography. Neuroimage 2003; 20:2040-2050.

23. Toga AW, Santori EM, Hazani R, Ambach
K. A 3 D digital map of rat brain. Brain Res Bull 1995; 38:77-85.

24. Belanger S, Ma W, Chabot JG, Quirion $R$. Expression of calcitonin gene-related peptide, substance $P$ and protein kinase $C$ in cultured dorsal root ganglion neurons following chronic exposure to $\mathrm{mu}$, delta and kappa opiates. Neuroscience 2002; 115:441-453.

25. Feng JT, Liu HQ, Xu JG, Gu YD, Shen YD. Differences in brain adaptive functional reorganization in right and left total brachial plexus injury patients. World Neurosurg 2015; 84:702-708.

26. Iwasaki R, Matsuura Y, Ohtori S, Suzuki T, Kuniyoshi K, Takahashi K. Activation of astrocytes and microglia in the $\mathrm{C}_{3}-\mathrm{T}_{4}$ dorsal horn by lower trunk avulsion in a rat model of neuropathic pain. J Hand Surg Am 2013; 38:841-846.

27. Wang L, Yuzhou L, Yingjie Z, Jie L, Xin $Z$. A new rat model of neuropathic pain: Complete brachial plexus avulsion. Neurosci Lett. 2015; 589:52-56.

28. Kobayashi T, Yamauchi K, Matsuura Y, Kuniyoshi K, Takahashi K, Ohtori $S$. The effects of generally administered anti-nerve growth factor receptor (p75NTR) antibody on pain-related behavior, dorsal root ganglia, and spinal glia activation in a rat model of brachial plexus avulsion. J Hand Surg Am 2015; 40:2017-2025.

29. Liu Y, Wang L, Meng C, Zhou Y, Lao J, Zhao X. A new model for the study of neuropathic pain after brachial plexus injury. Injury 2017; 48:253-261.

30. Zhao Y, Wu T. Histone deacetylase inhibition inhibits brachial plexus avulsioninduced neuropathic pain. Muscle Nerve 2018; 58:434-440.

31. Liu Y, Wang L, Lao J, Zhao X. Changes in microRNA expression in the brachial plexus avulsion model of neuropathic pain. Int J Mol Med 2018; 41:1509-1517.

32. Todd AJ. Neuronal circuitry for pain processing in the dorsal horn. Nat Rev Neurosci 2010;11:823-836.

33. Groh A, Krieger P, Mease RA, Henderson L. Acute and chronic pain processing in the thalamocortical system of humans and animal models. Neuroscience 2018; 387:58-71.

34. Sweitzer S, Martin D, Deleo JA. Intrathecal interleukin-1 receptor antagonist in combination with soluble tumor necrosis factor receptor exhibits 
an anti-allodynic action in a rat model of neuropathic pain. Neuroscience 2001; 103:529-539.

35. Ledeboer A, Sloane EM, Milligan ED, Frank MG, Mahony JH, Maier SF, Watkins LR. Minocycline attenuates mechanical allodynia and proinflammatory cytokine expression in rat models of pain facilitation. Pain 2005; 115:71-83.

36. Li YY, Zhang B, Yu KW, Li C, Xie HY, Bao WQ, Kong YY, Jiao FY, Guan YH, Bai YL. Effects of constraint-induced movement therapy on brain glucose metabolism in a rat model of cerebral ischemia: A micro PET/CT study. Int J Neurosci 2018; 128:736-745.

37. Kim JH, Shin SH, Lee YR, Lee HS, Chon JY, Sung CH, Hong SJ, Lee JY, Moon HS. Ultrasound-guided peripheral nerve stimulation for neuropathic pain after brachial plexus injury: Two case reports. J Anesth 2017; 31:1-5.

38. Garrison CJ, Dougherty PM, Kajander KC, Carlton SM. Staining of glial fibrillary acidic protein (GFAP) in lumbar spinal cord increases following a sciatic nerve constriction injury. Brain Res 1991; 565:1-7.

39. Matsuura Y, Iwakura N, Ohtori S, Suzuki T, Kuniyoshi K, Murakami K, Hiwatari R, Hashimoto K, Okamoto S, Shibayama $M$. The effect of anti-NGF receptor (p75 neurotrophin receptor) antibod- ies on nociceptive behavior and activation of spinal microglia in the rat brachial plexus avulsion model. Spine 2013; 38:E332-E338.

40. Jutzeler CR, Patrick F, Eveline $H$, Armin C, Kramer JLK. Neuropathic pain and functional reorganization in the primary sensorimotor cortex after spinal cord injury. J Pain 2015; 16:1256-1267.

41. Dosenovic $S$, Jelicic KA, Miljanovic $M$, Biocic M, Boric K, Cavar M, Markovina $N$, Vucic K, Puljak L. Interventions for neuropathic pain: An overview of systematic reviews. Anesth Analg 2017; 125:643-652.

42. Denk F, Crow M, Didangelos A, Lopes D, Mcmahon S. Persistent alterations in microglial enhancers in a model of chronic pain. Cell Rep 2016; 15:1771-1781.

43. Davis KD, Flor $H$, Greely HT, lannetti GD, Mackey S, Ploner M, Pustilnik A, Tracey I, Treede RD, Wager TD. Brain imaging tests for chronic pain: Medical, legal and ethical issues and recommendations. Nat Rev Neurol 2017; 13:624-638.

44. Peyron R. Functional brain imaging: What has it brought to our understanding of neuropathic pain? A special focus on allodynic pain mechanisms. Pain 2016; 157(Suppl 1):S67-S71.

45. Denk F, Mcmahon SB, Tracey I. Pain vulnerability: A neurobiological perspective. Nat Neurosci 2014; 17:192-200.
46. Yee JR, Kenkel W, Caccaviello JC, Gamber $K$, Simmons $P$, Nedelman $M$, Kulkarni P, Ferris CF. Identifying the integrated neural networks involved in capsaicin-induced pain using $F M R I$ in awake TRPV 1 knockout and wild-type rats. Front Syst Neurosci 2015; 9:15.

47. Shankar H, Hansen J, Thomas K. Phantom pain in a patient with brachial plexus avulsion injury. Pain Med 2015; 16:777-781.

48. Yen CT, Lu PL. Thalamus and pain. Acta Anaesthesiol Taiwan 2013; 51:73-80.

49. Nakata H, Sakamoto K, Kakigi R. Meditation reduces pain-related neural activity in the anterior cingulate cortex, insula, secondary somatosensory cortex, and thalamus. Front Psychol 2014; 5:1489.

50. Bilbao A, Falfán-Melgoza C, Leixner S, Becker R, Singaravelu SK, Sack M, Sartorius A, Spanagel R, Weber-Fahr W. Longitudinal structural and functional brain network alterations in a mouse model of neuropathic pain. Neuroscience 2018; 387:104-115.

51. Navarro X, Vivo M, Valero-Cabre A. Neural plasticity after peripheral nerve injury and regeneration. Prog Neurobiol 2007; 82:163-201.

52. Mohanty CB. Central plasticity in brachial plexus injury: A neural domino effect. World Neurosurg 2016; 86:22-24. 